\title{
Pazienti e medici a confronto nel Lazio in occasione dell'Incontro Regionale promosso da AIAF a Roma lo scorso 22 giugno 2019
}

Giornale di Tecniche Nefrologiche e Dialitiche 2019, Vol. 3I(3) 20I-203

(C) The Author(s) 2019

Article reuse guidelines:

sagepub.com/journals-permissions DOI: $10.1177 / 0394936219869186$ journals.sagepub.com/home/gtn

(S)AGE

\author{
Stefania Tobaldini
}

Un'intera giornata dedicata a pazienti, familiari, medici e operatori della salute del Lazio, riuniti tutti insieme per confrontarsi e approfondire la malattia di Fabry. 亡̀ l'incontro regionale medici-pazienti del Lazio che si è svolto il 22 giugno scorso a Roma organizzato da Aiaf Onlus. Una delle tappe del circuito di incontri regionali che Aiaf, l'associazione italiana di riferimento per $\mathrm{i}$ pazienti Anderson-Fabry, sta promuovendo in tutta la Penisola con il supporto del suo Comitato Scientifico, il cui obiettivo è di rappresentare un punto di incontro tra pazienti, medici specialisti dei vari Centri regionali, infermieri, ricercatori e tutti i portatori di interesse che si occupano in diversi ambiti di malattia di Fabry.

Il convegno si è aperto al mattino con due sessioni parallele: una dedicata ai pazienti e caregiver, con un workshop sui bisogni delle persone con malattie rare e il ruolo associativo, a cura di Laura Gentile - Psicologa Psicoterapeuta, e una dedicata ai medici, che si sono confrontati sugli aspetti gestionali e sulle criticità della presa in carico dei pazienti Fabry, con riferimento allo specifico contesto regionale.

Coordinato dai professori Francesco Franceschi e Andrea Frustaci, il workshop riservato ai medici ha ampliamente affrontato problematiche e punti critici legati alla Fabry. Entrando nel merito clinico, medico e gestionale dei vari aspetti della malattia, si è passati dalla presa in carico attraverso il PDTA - piano diagnostico terapeutico assistenziale - alla delicata fase di transizione dall'età pediatrica all'adulta, per focalizzare poi l'attenzione sulla questione dello screening neonatale, la terapia domiciliare e la necessità di istaurare un collegamento strutturato e capillare tra centri di riferimento e MMG e PLS, rispettivamente Medico di Medicina Generale e Pediatra di Libera Scelta.

Come illustrato dalla dott.ssa Federica Deodato, la presa in carico è il punto di arrivo di un percorso che va dalla conferma diagnostica al follow up, passando per l'inquadramento fenotipico e la terapia. Considerando la difficoltà di valutazione clinica della malattia lisosomiale, che si differenzia in forme severe, precoci e asintomatiche, è indispensabile trovare il momento giusto per iniziare il trattamento terapeutico, poiché aspettare il sintomo può comportare l'instaurarsi di importanti danni d'organo. Oggi è possibile su base istologia e non solo sintomatologica trovare un equilibrio tra tempestività e ospedalizzazione. Non va dimenticato che per anni la malattia di Fabry è stata circoscritta all'adulto, fin quando non è stato scoperto che il suo esordio è pediatrico. Proprio per questo la transizione tra le due età deve implicare un processo di comunicazione tra le equipe mediche del bambino e dell'adulto, a partire dal PDTA di supporto.

Lo screening neonatale esteso alla Malattia di Anderson-Fabry invece, trattato dalla dott.ssa Antonia Nucera, include un programma complesso, integrato e multidisciplinare basato su diagnosi e presa in carico familiare, che anche grazie ad un appello di Aiaf Onlus è stato inserito nell'ultima Legge di Bilancio per le patologie lisosomiali, anche se si stanno ancora attendendo i decreti attuativi.

Si è poi parlato di home therapy, con una discussione stimolata dalla relazione della dott.ssa Cristina Chimenti, nel corso della quale è stata sollevata la disomogeneità nazionale del servizio e le difficoltà nel coordinamento a livello regionale. La Regione Lazio ha adottato un'apposita delibera che consente la terapia domiciliare per i pazienti con Malattia di Anderson-Fabry, orientandosi (come accade in molte altre regioni italiane) verso la terapia domiciliare somministrata da infermieri di aziende private,

Presidente AIAF Onlus, Roma, Italy

\section{Corrispondenza:}

Stefania Tobaldini, Presidente AIAF Onlus, Roma, Italy.

E-mail: presidente@aiaf-onlus.org 
con costo supportato dalle Aziende Farmaceutiche produttrici dei farmaci.

Per i pazienti delle regioni attualmente non favorevoli alla terapia domiciliare, invece, il problema resta aperto. I pazienti Fabry infatti difficilmente riescono ad ottenere il riconoscimento dello stato di handicap in quanto spesso non hanno problemi di deambulazione e questo rende impossibile l'accesso all'Assistenza Domiciliare Integrata (ADI) del Servizio Sanitario Regionale. Questo comporta non pochi problemi ai pazienti, che sono costretti a ricevere la terapia in ospedale, in orari incompatibili con quelli lavorativi e con la necessità di attingere a permessi o ferie per potersi curare. Per risolvere l'attuale situazione disomogenea sul territorio nazionale, AIAF è attualmente impegnata in una campagna di sensibilizzazione nei confronti delle Istituzioni, insieme alle altre associazioni di pazienti con malattia lisosomiale.

La mattinata è proseguita con la riflessione della dott.ssa Daniela Pisani, che ha esposto la necessità di una presa in carico sempre più condivisa con il territorio, e in particolare con il coinvolgimento dei Medici di Medicina Generale e Pediatri di Libera Scelta e non solo del Centro di Riferimento, nella stesura di un piano assistenziale individualizzato, nella prospettiva di un modello ampio di competenze da mettere a sistema con il supporto della regione.

Nel pomeriggio, la Presidente dell'Intergruppo Parlamentare delle Malattie Rare, Senatrice Paola Binetti, è intervenuta ricordando quanto i due obiettivi di AIAF, la
Terapia Domiciliare in tutte le Regioni italiane e l'effettivo avvio dell'inserimento delle patologie lisosomiali nel programma e nel panel dello Screening Neonatale Esteso,

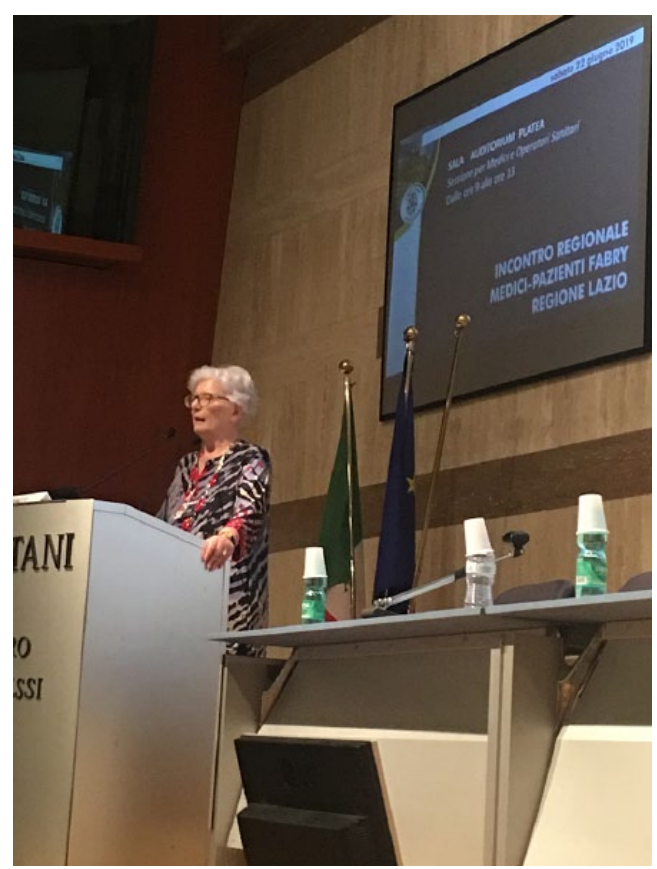

Fig. I La Presidente dell'Intergruppo Parlamentare per le Malattie Rare, Senatrice Paola Binetti.

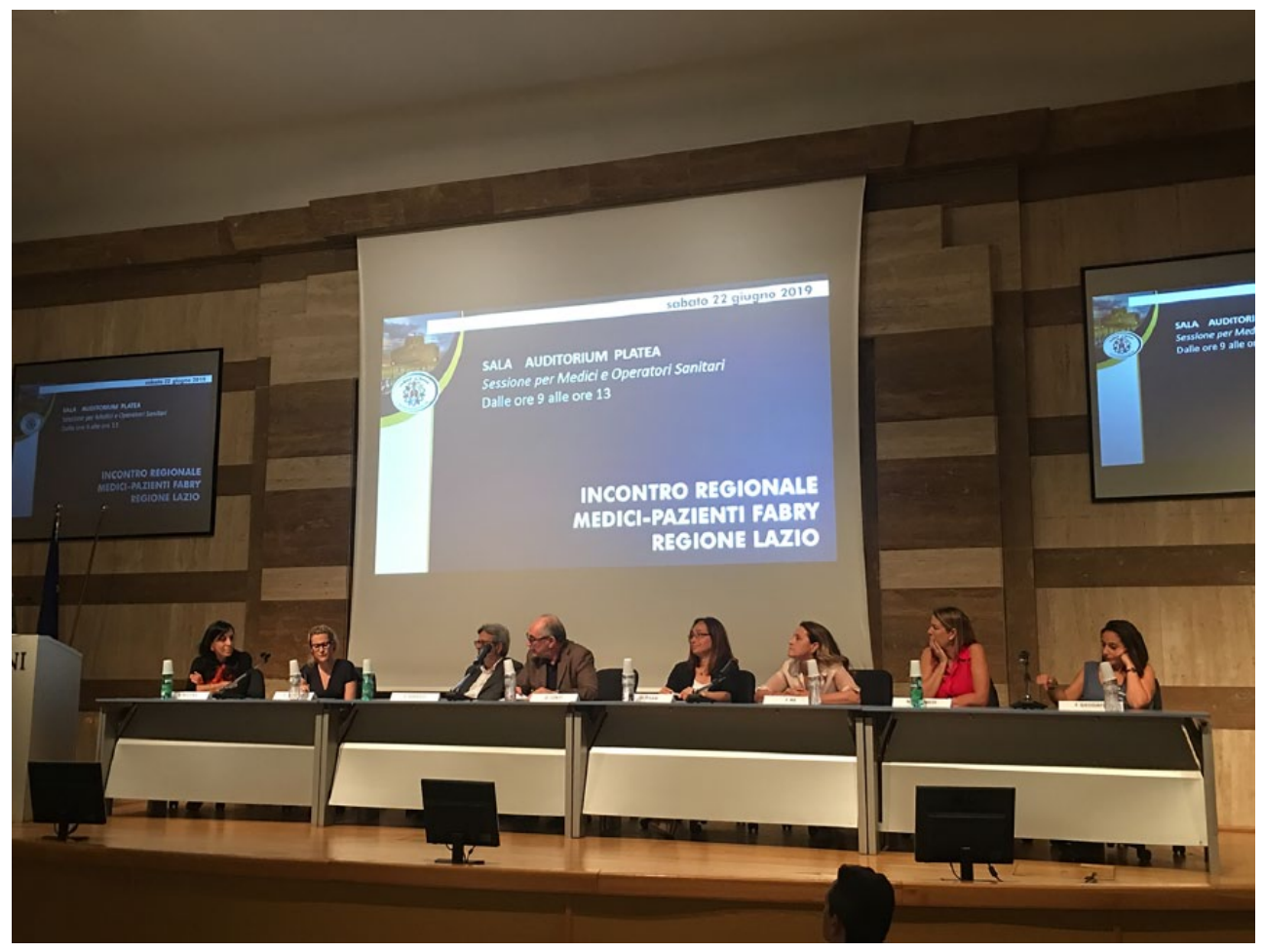

Fig. 2 Da sinistra: Dott.ssa Antonia Nucera, Dott.ssa Chiara Lanzillo, Prof. Sandro Feriozzi, Prof. Guido Conti, Dott.ssa Daniela Pisani, Dott.ssa Federica Re, Dott.ssa Marina Diomedi e Dott.ssa Federica Deodato. 


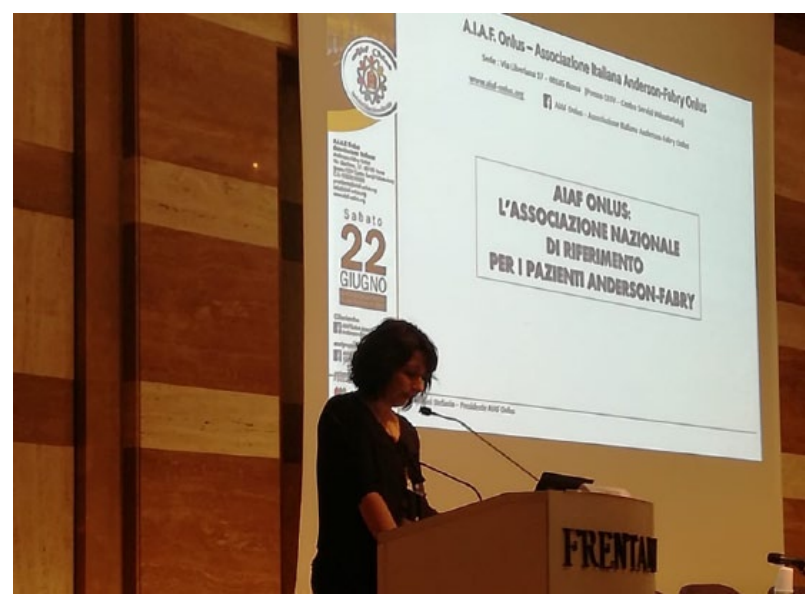

Fig. 3 Stefania Tobaldini, Presidente di AIAF Onlus.

siano legati al nuovo Piano Nazionale delle Malattie Rare. Questo, scaduto nel 2016, va adeguato alle nuove patologie e prestazioni, tenendo conto degli ERN, dei nuovi farmaci e della maturata sensibilità di associazioni e pazienti, sempre più esperti. Ha inoltre esortato a "non avere mai paura di alzare la voce attraverso le associazioni. Il diritto esiste ma a volte è difficile esigerlo, per questo serve la forza della rappresentatività". E ha proseguito "non sarete mai abbastanza insistenti, neanche quando avrete ottenuto, fin quando non arriverà sul tavolo del paziente."

La Dottoressa Esmeralda Castronuovo, della Direzione Regionale Salute ed Integrazione Socio Sanitaria della Regione Lazio, è intervenuta per illustrare le prassi organizzative e il sistema informatico regionale dedicato, un importante aiuto nella gestione del paziente.

Infine, in chiusura del convegno, la tavola rotonda con le domande dei pazienti ai medici presenti.

È stata per i pazienti del Lazio una preziosa occasione di conoscenza reciproca, ma anche di riflessione, racconto e confronto sulla relazione d'aiuto e sull'esperienza personale riguardante la malattia. La quantità degli stimoli emersi nei due workshop del mattino (pazienti da una parte e medici dall'altra) ci ha confermato l'importanza di creare occasioni di incontro, e rappresenta per noi uno stimolo a continuare a progettare nuovi incontri in altre regioni italiane, auspicando anche il coinvolgimento e la partecipazione dei Medici di Medicina Generale e Pediatri di Libera Scelta per poter meglio veicolare informazioni sulla Malattia di Anderson-Fabry anche tra chi non si occupa specificamente di questa malattia.

Per perseguire l'obiettivo di creare una rete di auto supporto tra i pazienti, è necessario il coinvolgimento attivo dei clinici di riferimento, affinchè $\mathrm{i}$ pazienti conoscano la realtà associativa e la possibilità di far parte di una comunità, che può essere un'utile risorsa per il paziente per superare la percezione di isolamento dovuta alla consapevolezza di dover convivere con una malattia rara come la malattia di Anderson-Fabry.

Gli incontri regionali medici-pazienti proseguiranno, con prossima tappa a Firenze, in Toscana, il 19 Ottobre 2019. Per informazioni e per partecipare scrivere a: presidente@aiaf-onlus.org.

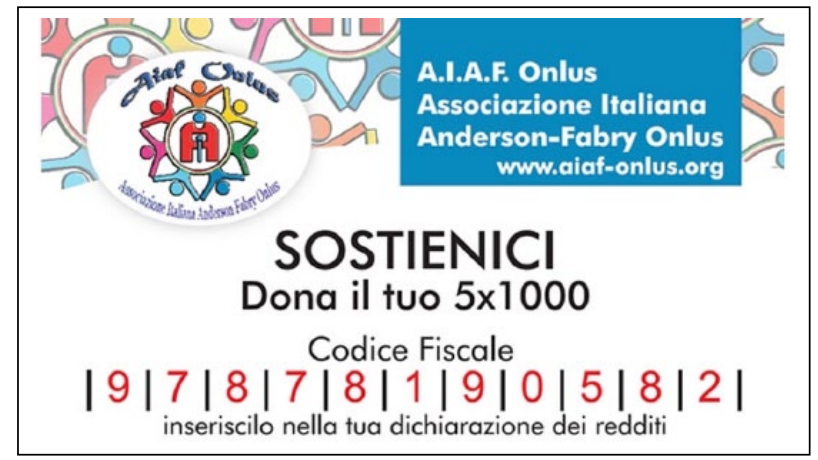

